\title{
Metal-insulator transition in the In/Si(111) surface
}

\author{
S. Riikonen ${ }^{\mathrm{a}, \mathrm{b}}$, A. Ayuela ${ }^{\mathrm{b}}$, D. Sánchez-Portal ${ }^{\mathrm{c}, \mathrm{b}}$ \\ a Departamento de Física de Materiales, Facultad de Química, Universidad del País Vasco, Apdo. 1072, 20080 \\ Donostia-San Sebastián, Spain \\ ${ }^{\mathrm{b}}$ Donostia International Physics Centre (DIPC), Paseo Manuel de Lardizabal 4, 20018 Donostia-San Sebastián, Spain \\ c Centro Mixto CSIC-UPV/EHU, "Unidad de Física de Materiales", Apdo. 1072, 20080 Donostia-San Sebastián, Spain
}

\begin{abstract}
The metal-insulator transition observed in the $\operatorname{In} / \mathrm{Si}(111)-4 \times 1$ reconstruction is studied by means of ab initio calculations of a simplified model of the surface. Different surface bands are identified and classified according to their origin and their response to several structural distortions. We support the, recently proposed [New J. of Phys. 7 (2005) 100], combination of a shear and a Peierls distortions as the origin of the metal-insulator transition. Our results also seem to favor an electronic driving force for the transition.
\end{abstract}

Quasi one-dimensional reconstructions formed by metal deposition on $\operatorname{Si}(111)$ have been intensively studied in recent years. The electronic correlations and the coupling between electronic and structural degrees of freedom are enhanced in one-dimension and, as a consequence, several electronic and structural phase transitons are observed in these systems as the temperature is decreased. A nice example of this behavior is found in the $\operatorname{In} / \mathrm{Si}(111)$ system, which exhibits a $4 \times 1 \rightarrow 4 \times 2 \rightarrow 8 \times 2$ structural transition accompanied by a metal-insulator electronic transition.

The room-temperature (RT) $4 \times 1$ structure of the $\mathrm{In} / \mathrm{Si}(111)$ surface is well established $[1,2]$. It consists of two neighboring zigzag In wires along the $[11 \overline{2}]$ direction. Each wire contains two In atoms per $4 \times 1$ cell and each In atom is bonded to one Si atom of the substrate. This model has been

\footnotetext{
Email addresses: swbriris@sc.ehu.es (S. Riikonen), swxayfea@sc.ehu.es (A. Ayuela), sqbsapod@sc.ehu.es ( D. Sánchez-Portal).
}

confirmed by ab-initio calculations $[3,4,5]$ which reproduce the scanning tunneling microscopy (STM) images [6,7], and the main features of the band structure.

At RT the system presents three metallic surface bands with similar dispersion [8]. However, when the temperature is lowered below 130 K [9] photoemission shows the formation of a band gap. This transition is accompanied with a doubling of the unit cell in the STM images [9]. The low temperature (LT) phase has been widely studied experimentally $[10,11,12,13]$. Although several results seem to favor a model where the indium wires suffer a strong dimerization and break-up into trimers, the question is still far from settled. In fact, most ab-initio calculations find metallic structures which are only slightly distorted with respect to the $\mathrm{RT}$ phase $[5,14]$. Thus, the driving force behind the combined metal-insulator and structural transition remains unclear.

Recently, an interesting mechanism for the 
gap opening has been proposed by Ahn et al. in Ref. [15]. The occupation of the surface bands in the $4 \times 1$ structure is quite close to two electrons. If one of these bands is depopulated (the upper one), the other two become very close to half-filled and thus are suitable to suffer a Peierls transition due to a periodicity doubling. If this is true, it seems to indicate that: $(i)$ there are at least two types of surface bands that originate in different regions of the substrate or have different symmetries, and (ii) the metal-insulator transition is the result of a combination of two distinct structural distortions that couple with different bands. This last point is consistent with the recent first-principles calculations by González et. al. [16]. These authors find an insulating $4 \times 2$ structure, reminiscent of that proposed by Kumpf et al. [1], as a result of a combined shear and Peierls distortion of the $4 \times 1$ RT phase.

The objective of the present work is to understand in detail the origin and characteristics of the different electronic states involved in the metalinsulator transition, and how they couple to different structural distortions. The emphasis is on the electronic bands associated with the indium atoms in the substrate. We use a simplified model (shown in Fig. 1) that captures the essence of the system. Our results support the main conclusions of Ref. [16], and point to a primary electronic origin of the structural transition. Of course, in the real surface we can expect a delicate competition between the gain of electronic energy and the elastic energy associated with the different distortions. Work along this line is in progress and will be published elsewhere [17].

Our calculations were performed with the SIESTA method [18]. We used the generalized gradient approximation [19] to the density functional theory, norm-conserving pseudopotentials [20], and a double- $\zeta$ polarized basis set of numerical atomic orbitals with cutoff radii corresponding to an energy shift of $200 \mathrm{meV}$ [18]. ${ }^{1}$ The density of the auxiliary real-space grid [18] was equivalent to a plane-wave cutoff of 100 Ry. We typically used 12

1 The cutoff radius was 4.95 a.u. for the $s$ and $p$ orbitals of $\mathrm{H}$; for In, $s, p$ and $d$ orbitals had cutoff radii of 5.95, 7.83 , and 7.83 a.u., respectively. inequivalent k-points along the wire direction (up to 250 in convergence tests, and 100 in the calculations shown in Fig. 4). We used periodic boundary conditions with a vacuum distance of $15 \AA$.

Figure 1 presents the simple model used here to study the electronic properties of indium wires in the In/Si(111) surface. We keep the two zigzag In wires present in the $4 \times 1$ unit cell, and substitute the neighboring silicon atoms with hydrogen. The In-H distances were optimized (1.86 $\AA$ ), and the relative positions of the In-H pairs were kept fixed for the rest of the study. This system provides a qualitative model that, however, retains the main features of the electronic band structure of the In/Si(111) surface (see below). The In-H bond is slightly more ionic than the In-Si bond. However, we have checked that saturating with $\mathrm{SiH}_{3}$ groups instead of $\mathrm{H}$ atoms leads to a very similar band structure.

Figure 2 shows the evolution of the band structure of our model as a function of the wire-wire distance $d$. There are two types of indium atoms in each wire, $\operatorname{In}(1)$ and $\operatorname{In}(2)$. The coordination of the $\operatorname{In}(2)$ atoms changes with $d$. Panel (a) corresponds to non-interacting (large $d$ ) zigzag wires, while panel (c) $(d=2.15 \AA)$ corresponds to a configuration similar to that found in the In/Si(111)- $4 \times 1$ reconstruction. Although not completely evident due to appearance of interaction gaps, the band structure in Fig. 2 (a) can be rationalized in terms of three bands: (I) a strongly dispersive band associated with the $\operatorname{In}(1)$ atoms and the $\operatorname{In}(2)-\mathrm{p}_{y}$ orbitals (circles), a flat band (II) with a clear contribution from $\operatorname{In}(1)$ and the $\operatorname{In}(2)-\mathrm{p}_{z}$ orbitals (triangles), and another flat band (III) with large $\operatorname{In}(2)$ $\mathrm{p}_{x}$ character (squares). Taken into account the hydrogen saturation, each In atom contributes with two valence electrons. Thus we have four electrons two distribute in these bands. Band (II) is doubly occupied and does not play any role in the argumentation below. Bands (I) and (III), however, are half-filled. Band (III) can be associated with the "dangling-bonds" in the $\operatorname{In}(2)$ atoms that project approximately into the $\mathrm{x}$-direction and the vacuum.

As $d$ is reduced, the interaction between the wires modifies the band structure. This can be seen in Fig. 2(b) and (c). Particularly, the dispersion of 
bands derived from band (III) largely increase as a result of the overlap of the dangling-bonds in the neighboring wires. Finally, the electronic states associated with this band become highly delocalized in the region between the two zigzag wires, and the band exhibits an almost free-electron dispersion. In the following we call this band the "interaction band".

Figure 2 (d) presents the band structure of the In/Si(111)- $4 \times 1$ reconstruction calculated with a slab containing four silicon bilayers [17]. Four surface bands can be located in the gap of the silicon substrate. Three of them cross the Fermi level with similar dispersions. This is qualitatively reproduced by our model. The agreement is improved if a small shift of the Fermi level is allowed (see Fig. 2 (c)). The information from our model allows to catalogue the surface bands in two types: (i) two bands that are derived from the band (I) of the right and left wires, and (ii) the interaction band. The first two bands have a larger weight inside each of the wires and are quite sensitive to the structure of the zigzag chains. In contrast, the interaction band is localized in the region between the two zigzag wires and thus is more influenced by the relative positions of the wires.

This division allows to envision a two-step route for the observed metal-insulator transition. Step one: the dimerization of the dangling-bonds from neighboring zigzag chains opens a gap in the interaction-band. This effect can be obtained without doubling the periodicity of the system, the relative displacement of the wires along their axes (see Fig. 3) suffices. Notice that this corresponds to the shear distortion in Ref. [16]. Step two: the remaining metallic bands need to accommodate two valence electrons. Since these two bands have very similar dispersions, both become approximately half-filled. As a consequence, the system is now suitable to suffer a Peierls transition. This two-step mechanism is consistent with the calculations of Ref. [16] and the experimental evidence in Ref. [15].

Figure 3 (a) shows the evolution of the energy and the band structure of our system as function of the shear distortion. One of the zigzag wires was displaced along the y-direction by a magnitude $\Delta y$ with respect to the other. For each dis- placement the distance between the wires $d$ was optimized. A gap is opened in the interaction band which widens with increasing $\Delta y$. For distortions larger than $\Delta y \sim 0.5 \AA$ the Fermi level enters in this gap. This is reflected in the behavior of the energy that starts to decrease at this point. This behavior translates in an energy barrier of $\sim 5 \mathrm{meV}$ per In atom. The system is then left with two metallic bands that cross the Fermi level at nearby points in reciprocal space. This is also the case for the real In $/ \mathrm{Si}(111)-4 \times 1$ surface as can be seen in Fig. 3 (b). However, in this case the behavior of the total energy is different. Although still in the range of a few $\mathrm{meV}$ per In atom for moderate distortions, the shear deformation always increases the energy of the system [17].

We now study the effect of doubling the periodicity along the wires. We consider a quite simple Peierls-like distortion: the length of one every four bonds is shorten (the undistorted bond length is $3.045 \AA$ ). The distortion is identical for both wires. Still we have four different possibilities according to the different relative locations of the distorted bonds in the neighboring wires. This is illustrated in Fig. 4 (a). While doubling the unit cell, the four distortions break the symmetry of the system in different ways. This detail is quite important. The distortions open a gap at $\frac{\pi}{2 a}$ due to the periodicity doubling. However, since the Fermi points do not exactly lie in that position, this does not guarantee that the system will become semiconducting. This is more clear for the extreme shear distortion $\left(\Delta y=2.15 \AA, \alpha=90^{\circ}\right)$. In this case there is a mirror plane parallel to the axis of the wires which is only preserved by "distortion 1". As a consequence of this symmetry, the band structure of the system submitted to the "distortion 1" presents a band crossing and the system remains metallic. For the other three distortions the band crossing is avoided due to the break of symmetry and a gap opens at the Fermi level. This can be appreciated in Fig. 4 (b). For $\alpha \neq 90^{\circ}$ the symmetry gap is always opened, although its magnitude depends again on each particular structural distortion. Figures 4 (b) and (c) present the total energy for the different Peierls-like distortions (a shear deformation has been previously applied to the system). "Distortion 2" and "distortion 4" are always the 
most favorables. It is worth noting that the structure proposed in Ref. [16] can be understood as the result of applying a combination of a shear distortion and the Peierls-like "distortion 2" presented above.

In summary, we have studied the electronic structure of the indium zigzag wires seen on the $\mathrm{In} / \mathrm{Si}(111)-4 \mathrm{x} 1$ reconstruction. The different surface bands are identified and classified according to their origin and their response to different structural distortions. We confirm that the combination of a shear and a Peierls distortion, proposed in Ref. [16], provides a reasonable and robust route for the observed metal-insulator transition in this system. Our results also point to an electronic driving force of this transition.

This work was supported by the Basque Dep. de Educación and the UPV/EHU, the Basque Dep. de Industria (project NANOMAT, ETORTEK program), the Spanish MEC, and the European Network of Excellence FP6-NoE "NANOQUANTA". S.R. also acknowledges support from the Magnus Ehrnroot foundation.

\section{References}

[1] O. Bunk, G. Falkenberg, J. H. Zeysing, L. Lottermoser, R. L. Johnson, Phys. Rev. B 59 (1999) 12228.

[2] G. Lee, S.-Y. Yu, H. Kim, J.-Y. Koo, H.-I. Lee, D. W. Moon, Phys. Rev. B 67 (2003) 35327.

[3] R. H. Miwa, G. P. Srivastava, Surf. Sci. 473 (2001) 123.

[4] J. Nakamura, S. Watanabe, M. Aono, Phys. Rev. B 63 (2001) 193307.

[5] J.-H. Cho, D.-H. Oh, K. S. Kim, L. Kleinman, Phys. Rev. B 64 (2001) 235302.

[6] J. Kraft, M. G. Ramsey, F. P. Netzer, Phys. Rev. B 55 (1997) 5384.

[7] A. A. Saranin, A. V. Zotov, K. V. Ignatovich, V. G. Lifshits, T. Numata, O. Kubo, H. Tani, M. Katayama, K. Oura, Phys. Rev. B 56 (1997) 1017.

[8] T. Abukawa, M. Sasaki, F. Hisamatsu, T. Goto, T. Kinoshita, A. Kakizaki, S. Kono, Surf. Sci. 325 (1995) 33.

[9] H. W. Yeom, S. Takeda, E. Rotenberg, I. Matsuda, K. Horikoshi, J. Schaefer, C. M. Lee, S. D. Kevan, T. Ohta, T. Nagao, S. Hasegawa, Phys. Rev. Lett. 82 (1999) 4898.
[10] C. Kumpf, O. Bunk, J. H. Zeysing, Y. Su, M. Nielsen, R. L. Johnson, R. Feidenhans'l, K. Bechgaard, Phys. Rev. Lett. 85 (2000) 4916.

[11] S. J. Park, H. W. Yeom, S. H. Min, D. H. Park, I.-W. Lyo, Phys. Rev. Lett. 93 (2004) 106402.

[12] S. Kurata, T. Yokoyama, Phys. Rev. B 71 (2005) 121306.

[13] K. Fleischer, S. Chandola, N. Esser, W. Richter, J. F. McGilp, Phys. Rev. B 67 (2003) 235318.

[14] J.-H. Cho, J.-Y. Lee, L. Kleinman, Phys. Rev. B 71 (2005) 81310.

[15] J. R. Ahn, J. H. Byun, H. Koh, E. Rotenberg, S. D. Kevan, H. W. Yeom, Phys. Rev. Lett. 93 (2004) 106401.

[16] C. González, J. Ortega, F. Flores, New J. of Phys. 7 (2005) 100.

[17] S. Riikonen, A. Ayuela, D. Sánchez-Portal, to be published.

[18] J. M. Soler, E. Artacho, J. D. Gale, A. Garca, J. Junquera, P. Ordejón, D. Sánchez-Portal, J. of Phys.: Condensed Matter 14 (2002) 2745.

[19] J. P. Perdew, K. Burke, M. Ernzerhorf, Phys. Rev. Lett. 77 (1996) 3865.

[20] N. Troullier, J. L. Martins, Phys. Rev. B 43 (1991) 1993.

[21] R. S. Mulliken, J. Chem. Phys. 23 (1955) 1841. 
Fig. 1. Our model of the In wires in the $\operatorname{In} / \operatorname{Si}(111)-4 \times 1$ surface: it contains two zigzag indium wires saturated with hydrogen.

Fig. 2. (a)-(c) Band structure (along the wires axes) of the system shown in Fig. 1 as a function of the wire-wire distance $d$ : (a) isolated zigzag In wire $(\mathrm{d}=10.8 \AA)$, (b) $\mathrm{d}=4.31 \AA$, and (c) $\mathrm{d}=2.15 \AA$. Different symbols indicate the distinct character of the bands as determined from a Mulliken population analysis [21]: $\operatorname{In}(1)$ and $\operatorname{In}(2)-\mathrm{p}_{y}$ (circles), $\operatorname{In}(2)-\mathrm{p}_{x}$ (squares), and $\operatorname{In}(1)$ and $\operatorname{In}(2)-\mathrm{p}_{z}$ (triangles). (d) Band structure of the $\mathrm{In} / \mathrm{Si}(111)-4 \times 1$ reconstruction along the $[11 \overline{2}]$ direction calculated with a slab containing four silicon bilayers [17], the bands with strong indium character are highlighted with circles. Energies are always referred to the Fermi level.

Fig. 3. Shear distortion. (a) Energy per indium atom as a function of the relative displacement $\Delta y$ of the indium wires along their axes (see the scheme in the upper part of panel (a)). The band structures for three different values of $\Delta y$ are also shown. (b) Band structure of the In $/ \mathrm{Si}(111)-4 \times 1$ reconstruction with $\Delta y=1.65 \AA$. The inset shows the Brillouin zone, $\Gamma \mathrm{X}$ and $\mathrm{YM}$ run along the In wires. Energies in the band structures are referred to the Fermi level.

Fig. 4. Peierls distortion. (a) Scheme showing four inequivalent Peierls-like distortions: the lenght of one of the bonds (indicated by an arrow) is modified by the same amount in both wires; different distortions correspond to different relative positions of the distorted bonds and are numbered according to the labels of the different bonds in the right wire. Panels (b) and (c) show the total energy per indium atom as a function of the modified bond length for distortions 1 (solid), 2 (dashed), 3 (dotted), 4 (dash-dotted). Panel (b) corresponds to $\alpha=90^{\circ}(\Delta y=2.15 \AA)$, while in panel (c) $\alpha=154^{\circ}(\Delta y=1.05 \AA)$. The insets show the band structures close to the Brillouin-zone boundary for distortions 1 and 2 in panel (b), and 2 in panel (c). Energies in the band structures are referred to the Fermi level. 
This figure "fig1.jpg" is available in "jpg" format from: http://arxiv.org/ps/cond-mat/0512613v1 
This figure "fig2.jpg" is available in "jpg" format from: http://arxiv.org/ps/cond-mat/0512613v1 
This figure "fig3.jpg" is available in "jpg" format from: http://arxiv.org/ps/cond-mat/0512613v1 
This figure "fig4.jpg" is available in "jpg" format from: http://arxiv.org/ps/cond-mat/0512613v1 